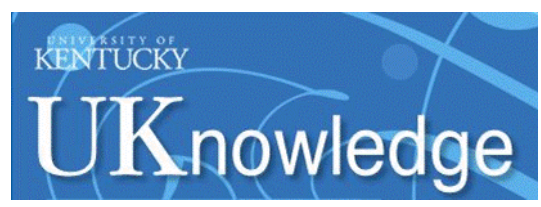

University of Kentucky

UKnowledge

\title{
An Investigation into the Effects of Luminosity on the Mid-Infrared Spectral Energy Distributions of Radio-Quiet Quasars
}

\author{
S. C. Gallagher \\ University of California \\ G. T. Richards \\ Johns Hopkins University \\ M. Lacy \\ California Institute of Technology \\ D. C. Hines \\ Space Science Institute \\ Moshe Elitzur \\ University of Kentucky, moshe@pa.uky.edu
}

See next page for additional authors

Follow this and additional works at: https://uknowledge.uky.edu/physastron_facpub

Part of the Astrophysics and Astronomy Commons, and the Physics Commons

Right click to open a feedback form in a new tab to let us know how this document benefits you.

\section{Repository Citation}

Gallagher, S. C.; Richards, G. T.; Lacy, M.; Hines, D. C.; Elitzur, Moshe; and Storrie-Lombardi, L. J., "An Investigation into the Effects of Luminosity on the Mid-Infrared Spectral Energy Distributions of RadioQuiet Quasars" (2007). Physics and Astronomy Faculty Publications. 202.

https://uknowledge.uky.edu/physastron_facpub/202

This Article is brought to you for free and open access by the Physics and Astronomy at UKnowledge. It has been accepted for inclusion in Physics and Astronomy Faculty Publications by an authorized administrator of UKnowledge. For more information, please contact UKnowledge@lsv.uky.edu. 


\section{An Investigation into the Effects of Luminosity on the Mid-Infrared Spectral Energy Distributions of Radio-Quiet Quasars}

Digital Object Identifier (DOI)

http://dx.doi.org/10.1086/513733

Notes/Citation Information

Published in The Astrophysical Journal, v. 661, no. 1, p. 30-37.

(c) 2007. The American Astronomical Society. All rights reserved.

The copyright holder has granted permission for posting the article here.

Authors

S. C. Gallagher, G. T. Richards, M. Lacy, D. C. Hines, Moshe Elitzur, and L. J. Storrie-Lombardi 
The Astrophysical Journal, 661:30 - 37, 2007 May 20

(C) 2007. The American Astronomical Society. All rights reserved. Printed in U.S.A.

\title{
AN INVESTIGATION INTO THE EFFECTS OF LUMINOSITY ON THE MID-INFRARED SPECTRAL ENERGY DISTRIBUTIONS OF RADIO-QUIET QUASARS
}

\author{
S. C. Gallagher, ${ }^{1}$ G. T. Richards,${ }^{2}$ M. Lacy, ${ }^{3}$ D. C. Hines,${ }^{4}$ M. Elitzur, ${ }^{5}$ and L. J. Storrie-Lombardi ${ }^{3}$ \\ Received 2006 August 28; accepted 2007 February 9
}

\begin{abstract}
We present an analysis of the effects of luminosity on the shape of the mid-infrared spectral energy distributions (SEDs) of 234 radio-quiet quasars originally presented by Richards et al. In quasars without evident dust extinction, the spectrally integrated optical and infrared luminosities are linearly correlated over nearly three decades in luminosity. We find a significant ( $299.99 \%$ confidence) correlation between the $1.8-8.0 \mu \mathrm{m}$ spectral index and infrared luminosity that indicates an enhancement of the mid-infrared continuum with increasing luminosity. Coupled with strong evidence for spectral curvature in more luminous quasars, we conclude that this trend is likely a manifestation of the "near-infrared (3-5 $\mu \mathrm{m}$ ) bump" noted in earlier quasar SED surveys. The strength of this feature is indicative of the contribution of emission from the hottest $(\gtrsim 1000 \mathrm{~K})$ dust to the mid-infrared spectrum; higher luminosity quasars tend to show more hot dust emission. Finally, the comparable distribution of bolometric corrections from the monochromatic $3 \mu \mathrm{m}$ luminosity as well as its lack of sensitivity to dust extinction as compared to the standard bolometric correction from $\nu L_{5100 \AA} \AA$ suggest that the former may be a more robust indicator of bolometric quasar luminosity. The close link between the power in the mid-infrared and optical and the effect of luminosity on the shape of the mid-infrared continuum indicate that considering mid-infrared emission independent of the properties of the quasar itself is inadequate for understanding the parsec-scale quasar environment.
\end{abstract}

Subject headings: galaxies: active — quasars: general

\section{INTRODUCTION}

Empirically, quasars are remarkable for spectral energy distributions (SEDs) with substantial power spanning many decades of frequency from the radio through the hard X-ray (e.g., Elvis et al. 1994). This SED encodes (although perhaps not unambiguously) the fundamental physical properties of the accreting black hole system such as the mass, spin, and accretion rate as well as random effects due to orientation; determining the mapping from the physical properties of interest to their spectral signatures is the ultimate goal of quasar SED studies.

The accretion flow near the black hole generates the vast majority of the continuum emission from the near-infrared through the hard X-rays, while infrared continuum light longward of $\sim 1 \mu \mathrm{m}$ is generally attributed to a parsec-scale, cold, and dusty region that is heated by and reprocesses the higher energy, direct continuum and serves to obscure the inner accretion system and broad-line region in type 2 (here defined as narrow-line) quasars. While the community generically refers to this medium as a "torus," this convenient picture merely accounts for the empirical evidence for axisymmetric dust obscuration without providing a coherent physical description of this structure (see Königl \& Kartje [1994] and Elitzur \& Shlosman [2006] for possible dynamical models). Regardless of the specific model, the quasar torus might be considered the interface between a quasar and its host galaxy, a reservoir for gas to fuel the black hole, and potentially a site of star formation. Understanding the nature of the torus is a primary aim of infrared quasar studies.

\footnotetext{
1 Department of Physics and Astronomy, University of California, Los Angeles, CA 90095-1547; sgall@astro.ucla.edu.

2 Department of Physics and Astronomy, Johns Hopkins University, Baltimore, MD 21218-2628.

3 Spitzer Science Center, California Institute of Technology, Pasadena, CA 91125.

${ }^{4}$ Space Science Institute, Boulder, CO 80301.

5 Department of Physics and Astronomy, University of Kentucky, Lexington, KY 40506-0055.
}

Richards et al. (2006a, hereafter R06a) recently presented $24 \mu \mathrm{m}$ through $u$-band photometric data of the largest sample to date of type 1 (broad-line) quasars. The resulting 259 SEDs, supplemented with archival Very Large Array, GALEX, and ROSAT data as available, span a bolometric luminosity range of approximately three decades. Overall, SEDs of type 1 quasars are remarkably similar out to $z=6$ (e.g., Shemmer et al. 2006; Hines et al. 2006; Jiang et al. 2006). However, in a qualitative comparison of two composite SEDs made from higher and lower luminosity objects, R06a noted subtle differences indicating that luminosity affected overall SED shapes, particularly in the mid-infrared (1.3$8 \mu \mathrm{m})$ as shown in Figure 1. Such differences in the mid-infrared spectra of quasars with higher mid-infrared fluxes were noted previously in the SEDs of a smaller sample of 35 optically selected quasars by Hatziminaoglou et al. (2005) and even earlier in an SED study of 29 Seyfert galaxies (Edelson \& Malkan 1986).

Luminosity is already known to affect some quasar emission properties. For example, the Baldwin effect characterizes the decreasing equivalent widths of ultraviolet emission lines with increasing ultraviolet continuum luminosity (e.g., Baldwin 1977; Richards et al. 2002; Shang et al. 2003). In addition, the maximum velocity of outflows seen in $\mathrm{C}$ IV absorption increases with ultraviolet luminosity (Laor \& Brandt 2002). Across broader spectral regions, the X-ray-to-optical flux ratio is known to decrease with increasing ultraviolet luminosity (e.g., Avni \& Tananbaum 1986; Strateva et al. 2005). Finally, and perhaps most relevant for the current study, the ratio of type 1 to type 2 quasars is found to increase with luminosity, as found in X-ray (e.g., Ueda et al. 2003; Akylas et al. 2006) and narrow emission-line surveys (Hao et al. 2005a). Overall, these empirical relationships indicate that luminosity affects the quasar system in some fashion.

In this paper, we investigate potential SED differences of type 1 quasars as a function of luminosity, focusing in particular on the rest-frame mid-infrared and optical/UV emission with the goal of understanding how the quasar affects its immediate environment. Throughout we assume a $\Lambda \mathrm{CDM}$ cosmology with 

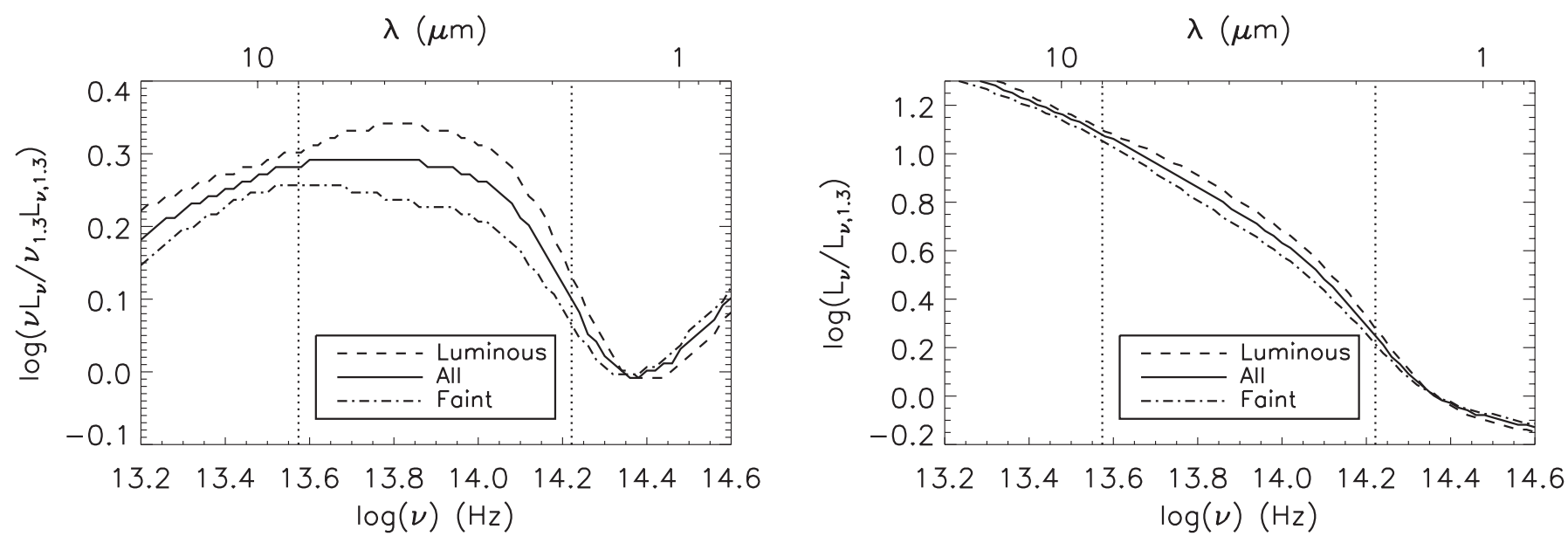

FIG. 1.-Plots of three SED composites from R06a made from the entire sample (solid curves), the luminous subset $\left[\log \left(L_{\mathrm{opt}}\right)>46.02\right.$ ergs s $\mathrm{s}^{-1}$; dashed curves], and the faint subset $\left[\log \left(L_{\mathrm{opt}}\right)<46.02 \mathrm{ergs} \mathrm{s}^{-1}\right.$; dot-dashed curves] in units of $\nu L_{\nu}$ (left), to emphasize the difference in spectral shapes, and $L_{\nu}($ right), to show the spectral region as we model it. The composite SEDs have been normalized to match at $1.3 \mu \mathrm{m}$. The SED resolution is a convolution of the IRAC (FWHM $=0.7,1.0,1.4$, and $2.9 \mu \mathrm{m}$ at 3.6, 4.5, 5.8, and $8.0 \mu \mathrm{m}$, respectively) and $24 \mu \mathrm{m}$ MIPS (FWHM $=5.3 \mu \mathrm{m}$ ) filter functions with the redshift distribution. Statistical subtraction of the host galaxy light is included; for detailed information on the composite construction, see R06a. For reference, 8 and $1.8 \mu \mathrm{m}$ are indicated with vertical dotted lines.

$\Omega_{M}=0.3, \Omega_{\Lambda}=0.7$, and $H_{0}=70 \mathrm{~km} \mathrm{~s}^{-1} \mathrm{Mpc}^{-1}$ (Spergel et al. 2003, 2007).

\section{ANALYSIS AND RESULTS}

We take as the starting point for our analysis the photometric SED data from Tables 1 and 2 of R06a, focusing on the spectral regions with complete coverage: the observed-frame optical and mid-infrared. The optical coverage is from the Sloan Digital Sky Survey (SDSS) ugriz photometric data (York et al. 2000) corrected for Galactic extinction. The mid-infrared data are from Spitzer Infrared Array Camera (IRAC) 3.6, 4.5, 5.8, and $8.0 \mu \mathrm{m}$ (full widths at half-maxima [FWHMs] of the spectral responses are $0.7,1.0,1.4$, and $2.9 \mu \mathrm{m}$, respectively) and the Multiband Imaging Photometer for Spitzer (MIPS) $24 \mu \mathrm{m}(\mathrm{FWHM}=5.3 \mu \mathrm{m})$ imaging. There has been no attempt to remove host galaxy light, which is likely to contribute most significantly to the SED at rest-frame $\sim 1 \mu \mathrm{m}$.

R06a included all SDSS quasars with publicly available Spitzer coverage as of the Data Release 3 quasar catalog of Schneider et al. (2005). The SDSS is quite complete to all but the reddest quasars to $i=19.1$ (Richards et al. 2006b); all SDSS quasars were detected by Spitzer. From the entire R06a sample of 259 quasars, those that are known to be radio luminous $\left[\log \left(L_{R}\right)>\right.$ $32 \mathrm{ergs} \mathrm{s}^{-1} \mathrm{~Hz}^{-1}$ at rest-frame $20 \mathrm{~cm}$, assuming $\left.\alpha_{\nu, R}=-1\right]$ or have no $24 \mu \mathrm{m}$ photometry have been eliminated. Note that the majority of R06a quasars (229/259) have only radio upper limits. Of these 229, 99 do not have sensitive enough flux limits to rule out high radio luminosity. The trimmed sample comprises 234 quasars with $z=0.14-5.21, \bar{z}=1.54 \pm 0.92, i=16.8-20.4$, $\bar{i}=18.9 \pm 0.7, M_{i}=-22.0$ to -28.2 , and $\bar{M}_{i}=-25.6 \pm 1.6$.

Throughout this section, when we refer to a linear fit, we are using the ordinary least-squares bisector method to determine the slope and intercept of a line fitted to (usually logarithmic) data values. Isobe et al. (1990) recommend this method for determining the functional relationship between ordinate and abscissa values when the errors are not well defined or insignificant. Errors quoted for the slopes are the standard deviations of the best-fitting values.

\subsection{Relative Integrated Infrared and Optical Luminosities}

As a first step, we investigated whether the relative power in the infrared versus the optical varies with luminosity. As midinfrared emission and optical photons are generated in physically distinct regions, there is no reason a priori why the relative power should (or should not) be affected by luminosity. Figure $2 a$ shows the logarithm of the ratio of infrared and optical luminosities versus the logarithm of the optical luminosity; the quantity $l_{\mathrm{IR}}-l_{\mathrm{opt}}$ may be considered a parameterization of the reprocessed to direct accretion-powered luminosity. An alternate depiction of the same data, $l_{\mathrm{IR}}$ versus $l_{\mathrm{opt}}$, is presented in Figure $2 b$. We use $l_{\mathrm{IR}}$ and $l_{\text {opt }}$ to indicate $\log \left(L_{\mathrm{IR}}\right)$ and $\log \left(L_{\mathrm{opt}}\right)$, respectively, with units of $\operatorname{ergs~s}^{-1}$; both values are taken from Table 1 of R06a. The first, $l_{\mathrm{IR}}$, is the luminosity integrated from $\log (\nu)=12.48$ to $14.48 \mathrm{~Hz}$ $(1-100 \mu \mathrm{m}) ; l_{\mathrm{opt}}$ is integrated from $\log (\nu)=14.48$ to $15.48 \mathrm{~Hz}$ $(0.1-1 \mu \mathrm{m})$. Power-law interpolation was used on the monochromatic luminosities calculated from the tabulated flux densities.

From visual inspection, there is a clear trend with increasing optical luminosity for quasars to have relatively more power in the optical, and a linear fit gives a slope of $-0.51 \pm 0.06(1 \sigma$ uncertainty). Nonparametric statistical tests of the bivariate data measuring Spearman's $\rho=-0.288$ and Kendall's $\tau=-0.194$ indicate that the probability of obtaining these values if the parameters are not correlated is $<1 \times 10^{-5}$; the statistical values and probabilities are listed in Table 1.

Given that $l_{\text {opt }}$ characterizes direct emission from the accretion disk, it may be considered in some sense a truer measure of overall quasar power than the indirect and reprocessed infrared light. A straightforward interpretation of this significant trend would be that a smaller fraction of the accretion-powered luminosity is reprocessed and emitted in the mid-infrared in more luminous quasars. However, optical photons are subject to dust reddening and extinction. The integrated optical luminosity measured by the observer would be reduced by extinction, while $l_{\mathrm{IR}}-l_{\mathrm{opt}}$ would increase in the same sense as the correlation. Therefore, if dust extinction is prevalent, it could induce the correlation seen in Figure $2 a$. As the small grains responsible for moderate optical extinction will not affect the infrared power (and may not in any case be along the same line of sight), $l_{\mathrm{IR}}$ may be a more robust measure of the total quasar power. Indeed, we perform the same statistical tests on $l_{\mathrm{IR}}-l_{\mathrm{opt}}$ versus $l_{\mathrm{IR}}$ and find that these two quantities are not correlated. The scatter about the mean is also roughly the same across almost three decades in $l_{\mathrm{IR}}$.

\subsection{Comparison of Optically Blue and Red Quasars}

The values of $l_{\mathrm{IR}}-l_{\text {opt }}$ evident in Figure $2 a$ span roughly one decade from -0.5 to 0.5 . As the apparent trend of $l_{\mathrm{IR}}-l_{\mathrm{opt}}$ versus 


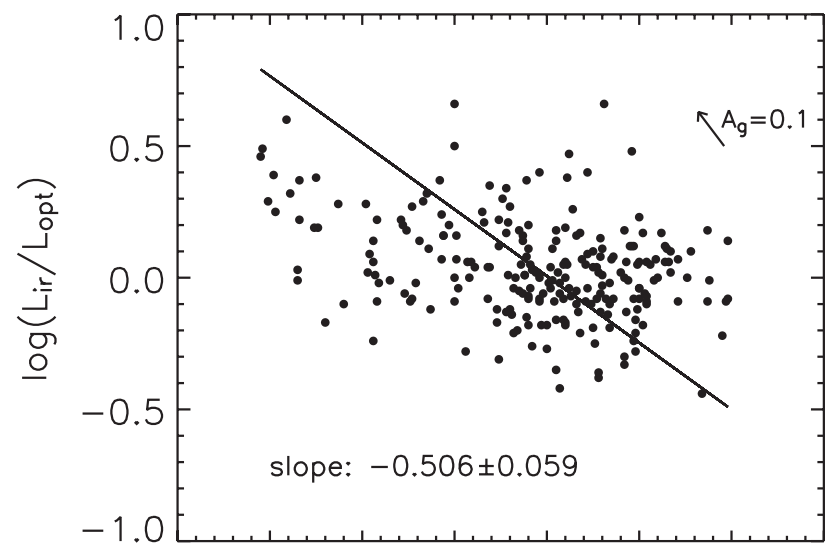

44.044 .545 .045 .546 .046 .547 .047 .5 $\log \left(L_{\text {opt }}\right)\left(\operatorname{erg~s}^{-1}\right)$

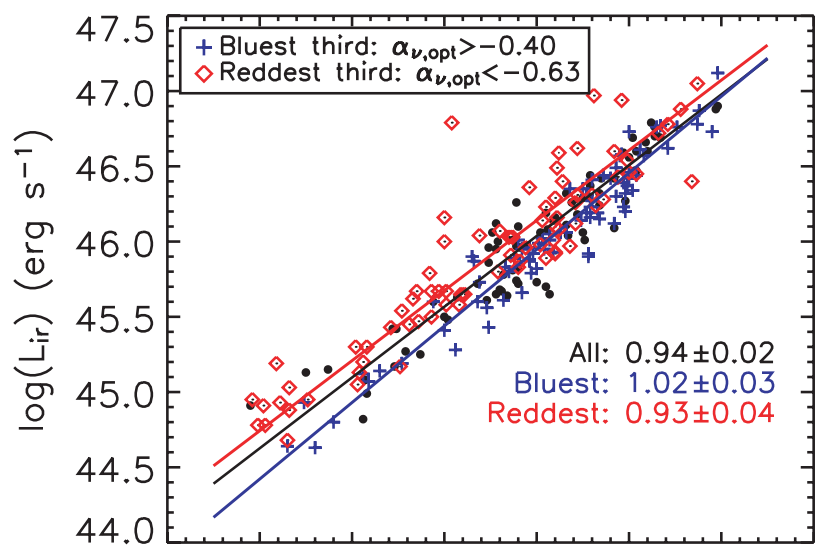

44.044 .545 .045 .546 .046 .547 .047 .5 $\log \left(L_{\text {opt }}\right)\left(\operatorname{erg~s}^{-1}\right)$

FIG. 2. - Left: Plot of $\log \left(L_{\mathrm{IR}} / L_{\mathrm{opt}}\right)$ vs. $\log \left(L_{\mathrm{opt}}\right)$ for the $234 \mathrm{SED}$ quasars. The solid line represents a linear fit to the plotted data; the corresponding slope and standard deviation are given in the figure. The labeled vector indicates the magnitude and direction of the effect of 0.1 mag of $g$-band extinction for SMC reddening of a power-law continuum with the median $\alpha_{\nu, \mathrm{opt}}=-0.49$. Right: Plot of $\log \left(L_{\mathrm{IR}}\right)$ vs. $\log \left(L_{\mathrm{opt}}\right)$ for the same sample. The optically bluest and reddest thirds are indicated with blue plus signs and red diamonds, respectively. Linear fits to all the data as well as the bluest and reddest thirds are drawn in solid lines; the best-fitting slopes are marked on the figure. While the left-hand panel indicates a significant correlation between the ratio of optical to infrared luminosity and the optical luminosity, an examination of the optically blue data implies that the data are consistent with $L_{\mathrm{IR}} \propto L_{\mathrm{opt}}$ if extinction is taken into account.

$l_{\text {opt }}$ plotted in Figure $2 a$ may be induced by extinction, we investigate whether extinction could also contribute to the width of the spread in $l_{\mathrm{IR}}-l_{\mathrm{opt}}$. To simply parameterize the color of the optical continuum, a power-law model $\left(l_{\nu} \propto \nu^{\alpha_{\nu, \text { opt }}}\right)$ was fit to the SDSS photometric data between rest-frame 1200 and $5000 \AA$. This region of the spectrum is typically dominated by the quasar, and by inspection of the continuum fits compared to the data (2-5 points in this region); a power-law model is appropriate for this purpose. For illustration, three sample SEDs are shown in Figure 3. From analysis of the SDSS composite quasar spectrum, the host galaxy contribution reddens the optical continuum slope for $\lambda>5000 \AA$ (Vanden Berk et al. 2001). Wavelengths $<1200 \AA$ are to be avoided, as the intervening Ly $\alpha$ forest reduces the flux significantly.

A histogram of $\alpha_{\nu \text {, opt }}$ values is shown in Figure $4 a$. At the blue (more positive) end, the distribution is roughly Gaussian; a long tail extends to the red (negative) end. The qualitative shape of this $\alpha_{\nu, \text { opt }}$ distribution is consistent with that of $\Delta(g-i)$, the SDSS $g-i$ color minus the mode of the $g-i$ color of quasars at the same redshift (Richards et al. 2003). The very red tail in the $\Delta(g-i)$ distribution is associated with continuum dust reddening, although intrinsically red quasars may contribute somewhat. To illustrate the effect of optical extinction on Figure $2 a$, a reddening vector has been drawn. The vector reflects the decrease in $l_{\mathrm{opt}}$ of $A_{g}=0.1$ mag of extinction, assuming Small Magellanic Cloud (SMC) reddening (as appropriate for quasars; e.g., Hopkins et al. 2004) of an $\alpha_{\nu \text {, opt }}=-0.49$ optical continuum integrated from 1000 to $10000 \AA$ (following R06a).

We use the median $\alpha_{\nu, \mathrm{opt}}=-0.49$ to divide the sample into "optically red" $\left(\alpha_{\nu, \text { opt }}<-0.49\right)$ and "optically blue" $\left(\alpha_{\nu, \text { opt }} \geq\right.$ $-0.49)$ groups. A comparison of the histograms of $l_{\mathrm{IR}}-l_{\mathrm{opt}}$ for the groups (Fig. 4b) shows an offset. A Student's $t$-test of the two populations indicates that the probability that they have the same mean is $<1 \times 10^{-6}$. This mean reduction in $l_{\mathrm{IR}}-l_{\mathrm{opt}}$ for the red

TABLE 1

Results from Nonparametric Bivariate Statistical Tests

\begin{tabular}{|c|c|c|c|c|}
\hline \multirow{2}{*}{$\begin{array}{c}\text { VARIABLES } \\
\text { INDEPENDENT/DEPENDENT (SAMPLE) }{ }^{\mathrm{a}}\end{array}$} & \multicolumn{2}{|c|}{ SPEARMAN } & \multicolumn{2}{|c|}{ Kendall } \\
\hline & $\rho$ & Probability $^{\mathrm{b}}$ & $\tau$ & Probability ${ }^{\mathrm{b}}$ \\
\hline$l_{\mathrm{opt}} /\left(l_{\mathrm{IR}}-l_{\mathrm{opt}}\right)(234)$ & -0.288 & $7.52 \mathrm{E}-6$ & -0.194 & $9.66 \mathrm{E}-6$ \\
\hline 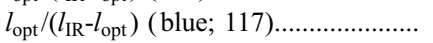 & -0.077 & $4.09 \mathrm{E}-1$ & -0.052 & $4.08 \mathrm{E}-1$ \\
\hline 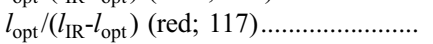 & -0.382 & $2.18 \mathrm{E}-5$ & -0.260 & $3.24 \mathrm{E}-5$ \\
\hline 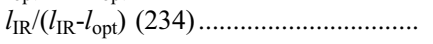 & 0.039 & $5.51 \mathrm{E}-1$ & 0.032 & $4.66 \mathrm{E}-1$ \\
\hline 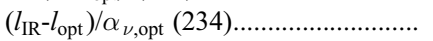 & -0.422 & $<1 \mathrm{E}-7$ & -0.292 & $<1 \mathrm{E}-7$ \\
\hline$l_{\mathrm{IR}} / \alpha_{\nu, \mathrm{IR}}(226) \ldots \ldots$ & -0.262 & $6.89 \mathrm{E}-5$ & -0.188 & $2.67 \mathrm{E}-5$ \\
\hline$l_{\mathrm{IR}} / \alpha_{\nu, \mathrm{IR}}(z=1.0-1.5 ; 55) \ldots \ldots \ldots \ldots \ldots \ldots$ & -0.508 & $7.48 \mathrm{E}-5$ & -0.375 & $5.36 \mathrm{E}-5$ \\
\hline$z / \alpha_{\nu, \mathrm{IR}}(226) \ldots \ldots \ldots$ & -0.058 & $3.88 \mathrm{E}-1$ & -0.061 & $1.74 \mathrm{E}-1$ \\
\hline 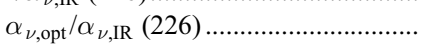 & 0.020 & $7.64 \mathrm{E}-1$ & 0.013 & $7.67 \mathrm{E}-1$ \\
\hline 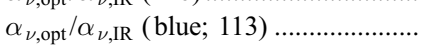 & 0.126 & $1.84 \mathrm{E}-1$ & 0.088 & $1.68 \mathrm{E}-1$ \\
\hline$l_{3 \mu \mathrm{m}} /\left(l_{3 \mu \mathrm{m}}-l_{5000} \AA\right)(234) \ldots \ldots \ldots \ldots \ldots$ & 0.074 & $2.62 \mathrm{E}-1$ & 0.047 & $2.85 \mathrm{E}-1$ \\
\hline$l_{3 \mu \mathrm{m}} /\left(l_{3 \mu \mathrm{m}}-l_{5000 \AA}\right)($ blue; 117)..... & 0.235 & $1.07 \mathrm{E}-2$ & 0.159 & $1.11 \mathrm{E}-2$ \\
\hline 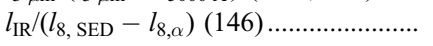 & -0.337 & $3.17 \mathrm{E}-5$ & -0.224 & $5.90 \mathrm{E}-5$ \\
\hline
\end{tabular}

a The sample labels and numbers of data points are given in parentheses. "Blue" and "red" refer to $\alpha_{\nu, \text { opt }} \geq-0.49$ and $\alpha_{\nu, \text { opt }}<-0.49$, respectively.

$\mathrm{b}$ The two-sided probability that the given variables are not correlated. 


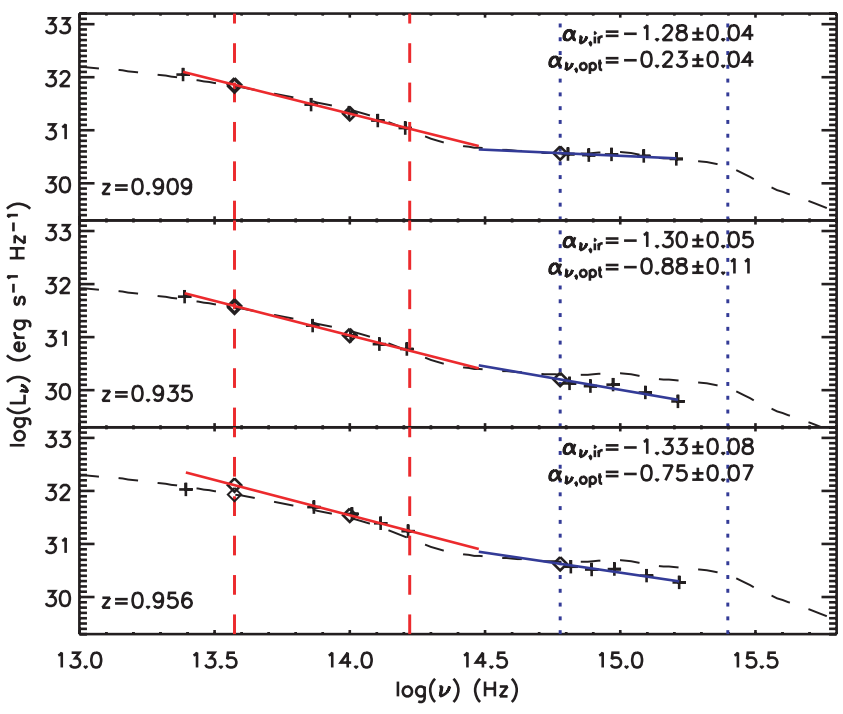

FIG. 3.-Sample SEDs illustrating the data analysis process; the abscissa is rest-frame frequency. Data are labeled with plus signs; thick open diamonds indicate the computed quantities (from left to right) of $L_{8, \alpha}, L_{3 \mu \mathrm{m}}$, and $L_{5000} \AA$ (see $\S 2.3$ for definitions). The thin dashed curve shows the mean composite SED from R06a normalized to the photometric data within 0.3 dex of rest-frame $8 \mu \mathrm{m}$; the thin open diamond represents $L_{8, \text { SED }}$ (overlapping with $L_{8, \alpha}$ in the top two panels). Red dashed and blue dotted vertical lines mark the boundaries of the mid-infrared and UV/optical fitting regimes, respectively. Solid lines are the best-fitting power-law models to the mid-infrared and optical data; they have been extrapolated to cover all of the data. The top panel is from a quasar with data closely matching the composite SED. The middle example shows a red (likely from dust reddening - note the decreased $L_{\mathrm{opt}}$ ) optical continuum, while the bottom panel indicates a measurable discrepancy between $L_{8, \text { SED }}$ and $L_{8, \alpha}$ indicating mid-infrared spectral curvature.

quasars is consistent with the expectation from extinction. In fact, within the optically red quasars, the subset of clearly dustreddened $\left(\alpha_{\nu \text {, opt }}<-0.70\right)$ quasars show the most extreme values of $l_{\mathrm{IR}}-l_{\mathrm{opt}}$.

Therefore, we conclude that optical extinction makes a significant contribution to the width in the $l_{\mathrm{IR}}-l_{\mathrm{opt}}$ distribution, and likely induces the correlation of $l_{\mathrm{IR}}-l_{\mathrm{opt}}$ versus $l_{\mathrm{opt}}$. In fact, this correlation disappears when only blue quasars are considered (see Table 1). For optically blue quasars, arguably the least likely to suffer from dust reddening, the data are consistent with a constant ratio of infrared to optical luminosity over approximately three decades in luminosity: $L_{\mathrm{IR}} \propto L_{\text {opt }}$ (see Fig. $2 b$ ).

\subsection{Dependence of the Mid-Infrared SED on Luminosity}

A close-up of the composite SEDs from R06a in the spectral region from 1 to $12 \mu \mathrm{m}$ reveals distinctions between the luminous and faint quasar SEDs (Fig. 1). When the SEDs are all normalized to $1.3 \mu \mathrm{m}$, the luminous SED shows enhanced flux in the midinfrared spectral region relative to the mean and faint composites. The three SEDs then almost meet again for $\lambda \gtrsim 10 \mu \mathrm{m}$. Note that these composites were constructed from broadband photometry, and therefore do not reflect interesting mid-infrared features such as strong $10 \mu \mathrm{m}$ silicate emission recently seen in several quasar spectra (e.g., Hao et al. 2005b; Siebenmorgen et al. 2005).

To investigate whether these differences are evident trends in all of the data rather than just in the composites, power-law fits have been performed to the Spitzer data in the rest-frame 1.8-8 $\mu \mathrm{m}$ region. As available, $15 \mu \mathrm{m} I S O$ and $J H K$ photometry were also incorporated. This spectral region was chosen to maximize the photometric coverage while reducing to the extent possible contamination from the host galaxy (which peaks in the near-infrared) and silicate emission (at $\sim 10 \mu \mathrm{m}$ ). Even in logarithmic units, curvature is evident in this spectral region (Fig. 1), and so the spectral index $\alpha_{\nu \text {, IR }}\left(l_{\nu} \propto \nu^{\alpha, \text { IR }}\right)$ should only be considered a simple parameterization of the data. Sample SEDs and the corresponding fits are shown in Figure 3. The value of $\alpha_{\nu \text {, IR }}$ for eight quasars with $z>3.5$ could not be measured because the MIPS $24 \mu \mathrm{m}$ data point was the only one in the bandpass.

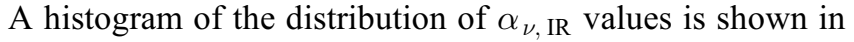
Figure $4 a$. For reference, the mean and standard deviation of the $\alpha_{\nu, \text { IR }}$ values, $-1.20 \pm 0.34$, are consistent with the $1-10 \mu \mathrm{m}$ spectral indices measured by Haas et al. (2003) of $-1.3 \pm 0.3$ for 64 Palomar-Green quasars observed with ISO.
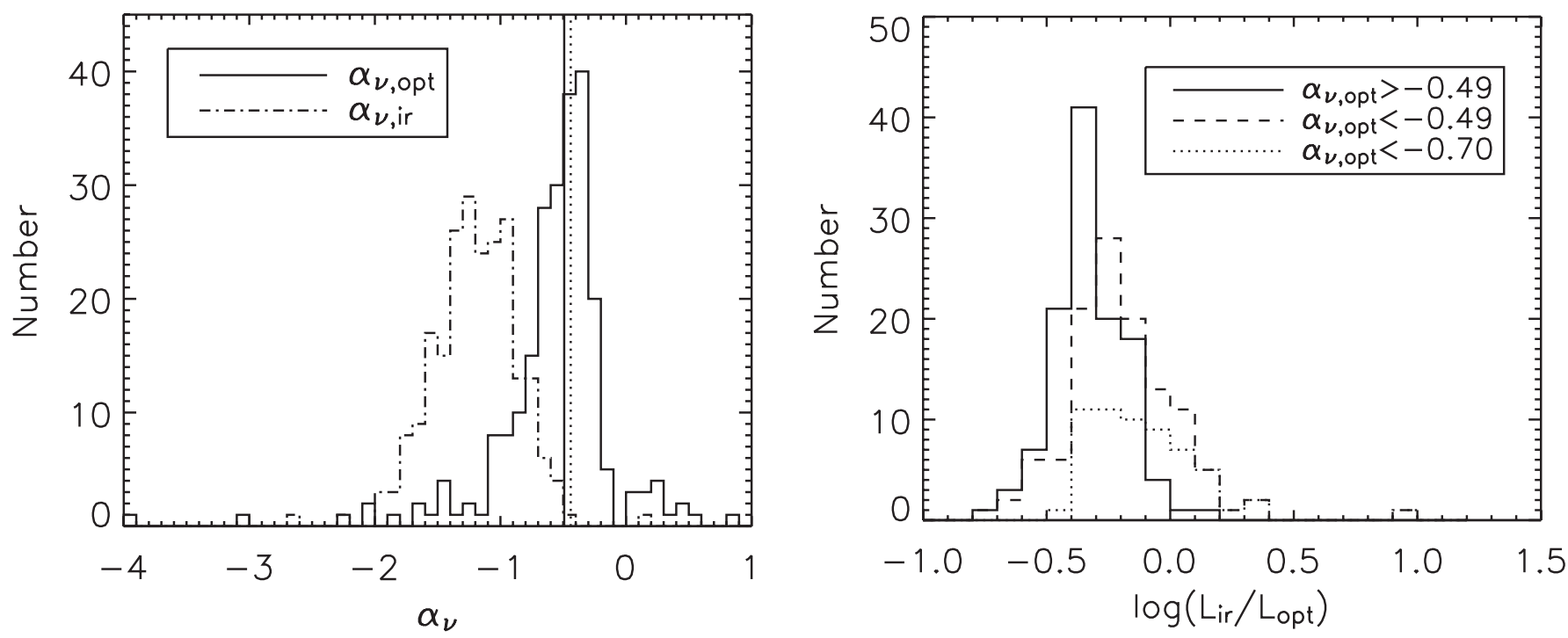

FIG. 4. - Left: Histograms of $\alpha_{\nu, \text { opt }}$ (solid) and $\alpha_{\nu, \text { IR }}$ (dot-dashed) for the SED sample. The $\alpha_{\nu}$ values are determined from power-law fits $\left(l_{\nu} \propto \nu^{\alpha_{\nu}}\right)$ to the photometric data; bluer continua are to the right. The median value for $\alpha_{\nu \text { opt }}$ of -0.49 is indicated with a solid vertical line. For reference, $\alpha_{\nu, \text { opt }}=-0.44$, the best-fit value to the continuum of the composite SDSS quasar spectrum, is also plotted (dotted vertical line; Vanden Berk et al. 2001). Right: Histograms of the distribution of log $\left(L_{\mathrm{IR}} / l_{\mathrm{opt}}\right.$ ) for the optically blue $\left(\alpha_{\nu \text { opt }} \geq-0.49\right.$; solid $)$ and red $\left(\alpha_{\nu, \text { opt }}<-0.49\right.$; dashed $)$ quasar populations. Quasars that are very likely to be dust reddened $\left(\alpha_{\nu, \text { opt }}<-0.70\right.$; dotted $)$ are also shown. The difference between these histograms indicates that at least some of the spread in the overall $\log \left(L_{\mathrm{IR}} / L_{\mathrm{opt}}\right)$ distribution is an artifact of optical extinction that reduces $L_{\mathrm{opt}}$. 


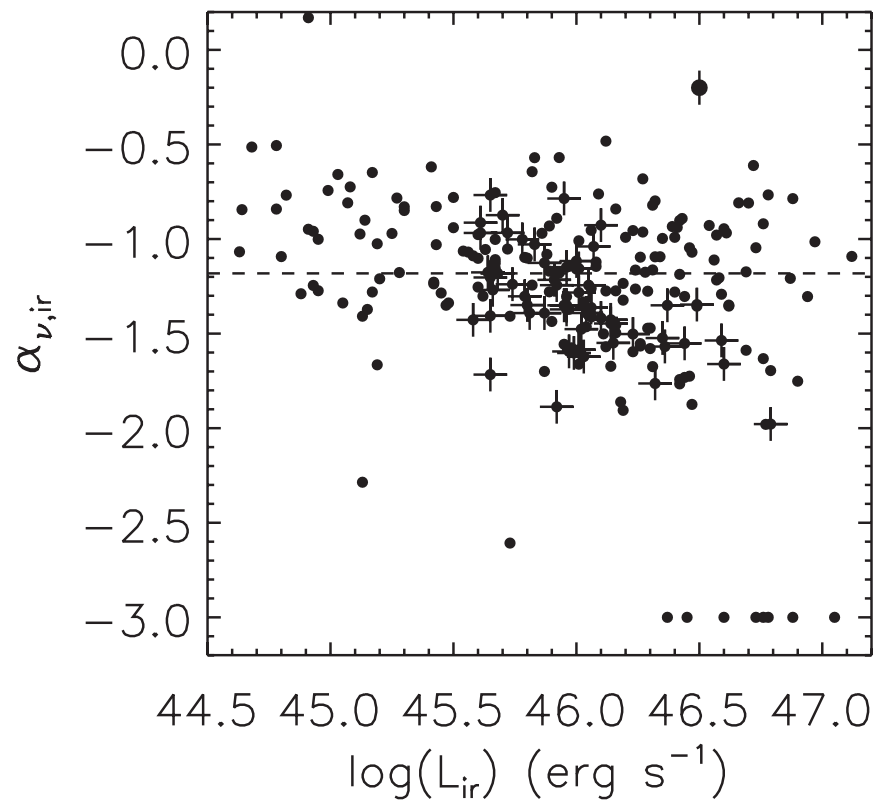

FIG. 5.- Infrared spectral index, $\alpha_{\nu \text {, IR }}$, vs. $\log \left(L_{\mathrm{IR}}\right)$ for the SED sample ( filled circles); the subset of the SED sample with $z=1.0-1.5$ is also marked with plus signs. The parameter $\alpha_{\nu \text {, IR }}$ is determined from power-law fits $\left(l_{\nu} \propto \nu^{\alpha, \mathrm{IR}}\right)$ to the Spitzer photometry between rest-frame 1.8 and $8.0 \mu \mathrm{m}$; the eight quasars at $z>3.5$ (plotted at $\alpha_{\nu \text { IR }}=-3.0$ ) only had one photometric data point in the bandpass; they are not included in the analysis. For reference, a horizontal dashed line marks

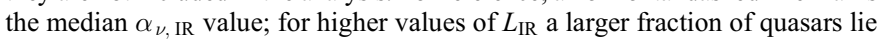
below the median. The median uncertainty in $\alpha_{\nu \text {, IR }}$ from fitting a power-law model to the photometry is indicated as a vertical error bar on a large, filled circle in the upper right corner of the plot.

As anticipated from the composite spectral results, the distribution of $\alpha_{\nu \text { IR }}$ versus $l_{\mathrm{IR}}$ shows a clear trend with increasing $l_{\mathrm{IR}}$ whereby the more luminous quasars tend to have steeper (more negative) spectral indices in the mid-infrared; this is evident in Figure 5. This apparent correlation is supported with the bivariate Spearman and Kendall tests, which give probabilities that $\alpha_{\nu \text { IR }}$ is not correlated with $l_{\mathrm{IR}}$ of $6.9 \times 10^{-5}$ and $2.7 \times 10^{-5}$, respectively. (We use $l_{\mathrm{IR}}$ instead of $l_{\mathrm{opt}}$ to avoid the problems of underestimating the luminosity due to extinction.)

Next, we investigate whether quasars are also brighter at 1.8$8 \mu \mathrm{m}$ relative to the optical continuum region. We choose to measure the monochromatic luminosities at $3 \mu \mathrm{m}$ and $5000 \AA\left(l_{3 \mu \mathrm{m}}\right.$ and $l_{5000 \AA}$ in logarithmic units) to represent the mid-infrared and optical, respectively: $3 \mu \mathrm{m}$ is near the middle of the $1.8-8.0 \mu \mathrm{m}$ bandpass, has good photometric coverage, and should have minimal contribution from even a star-forming host galaxy; $5000 \AA$ marks the red end of the quasar-dominated optical/UV continuum region. As seen from the results in Table $1, l_{3 \mu \mathrm{m}}-l_{5000} \AA$ does not change significantly as $l_{3 \mu \mathrm{m}}$ increases. If only optically blue quasars are considered (to reduce the potential effects of extinction), there is still no significant evidence for any change in $l_{3 \mu \mathrm{m}}-l_{5000} \AA$ for increasing $l_{3 \mu \mathrm{m}}$. The steepening of the mid-infrared continuum evident from $\alpha_{\nu \text {, IR versus }} l_{\mathrm{IR}}$ is much clearer evidence of midinfrared changes.

In the course of the previous tests, we visually examined each of the best-fitting optical and mid-infrared power-law fits in comparison with all of the SDSS and Spitzer photometry (cf. Fig. 3). Perhaps surprisingly, up to $z \sim 1.2$, extrapolating a simple powerlaw fit from the IRAC photometry predicts quite well $L_{\nu}$ in the MIPS $24 \mu \mathrm{m}$ bandpass. From $z=1.2$ to 2 , however, the extrapolation typically overpredicts $L_{\nu}$ in the MIPS $24 \mu \mathrm{m}$ bandpass. (Beyond $z=2$, the MIPS data point is included in the power-law

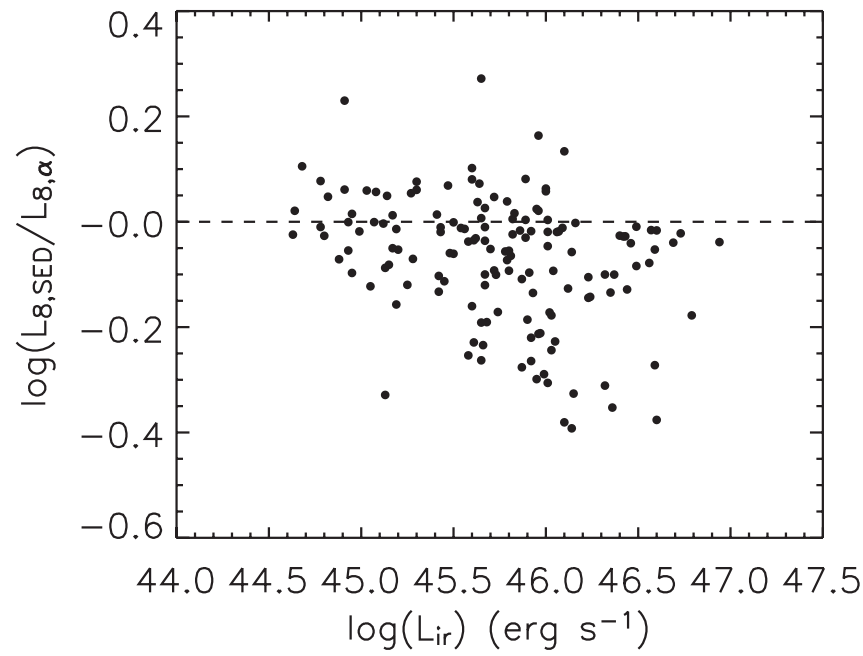

FIG. 6.-Logarithmic ratio of $L_{8}$, SED $\left(L_{8} \mu \mathrm{m}\right.$ determined from the R06a mean composite SED normalized to photometry within 0.3 dex of rest-frame $8 \mu \mathrm{m}$ ) to $L_{8, \alpha}\left(L_{8} \mu \mathrm{m}\right.$ calculated by extrapolating to $8 \mu \mathrm{m}$ from the mid-infrared power-law model) vs. $\log \left(L_{\mathrm{IR}}\right)$. For reference, a horizontal dashed line marks a ratio of unity. The strong decrease in $L_{8, \mathrm{SED}} / L_{8, \alpha}$ with increasing $L_{\mathrm{IR}}$ indicates increasing spectral curvature at higher luminosities.

fit.) This systematic effect is not because of a contribution of significant $10 \mu \mathrm{m}$ flux from silicate emission, which would cause the opposite effect. Instead, the deficit of $24 \mu \mathrm{m}$ emission is evidence for curvature in the mid-infrared spectrum. This is consistent with the luminous and faint composite SEDs, which when normalized at $1.3 \mu \mathrm{m}$ almost meet again near $10 \mu \mathrm{m}$ (see Fig. 1 and R06a).

To characterize spectral curvature, we calculate the monochromatic $8 \mu \mathrm{m}$ luminosity, $L_{8} \mu \mathrm{m}$, in two ways. For the first, $L_{8, \alpha}$, the best-fitting mid-infrared power-law spectral index and normalization are used to extrapolate to rest-frame $8 \mu \mathrm{m}$. For the second, $L_{8 \text {, SED }}$, photometric data points within 0.3 dex of rest-frame $8 \mu \mathrm{m}$ are used to normalize the R06a composite SED to measure the $8 \mu \mathrm{m}$ luminosity. The bottom panel in Figure 3 illustrates the discrepancy between $L_{8, \text { SED }}$ and $L_{8, \alpha}$ seen in some objects. Quasars without at least three data points in the $1.8-8.0 \mu \mathrm{m}$ regime and photometry within 0.3 dex of $8 \mu \mathrm{m}$ were eliminated from this analysis to mitigate redshift effects; the resulting sample has $146 \mathrm{ob}-$ jects. As seen in Figure 6, the strong decrease in $L_{8, \mathrm{SED}} / L_{8, \alpha}$ with increasing $L_{\mathrm{IR}}$ is indicative of significant curvature in the spectrum (see Table 1).

Overall, we find strong evidence for significant differences in the mid-infrared spectral regime as a function of luminosity. Specifically, more luminous quasars show steeper (more negative) values of $\alpha_{\nu \text {, IR }}$. Extrapolating $\alpha_{\nu \text {, IR }}$ to rest-frame $8 \mu \mathrm{m}$ also overpredicts the $8 \mu \mathrm{m}$ luminosity in luminous quasars, thus demonstrating spectral curvature. Both these results are consistent with the expectation from the luminous composite shown in Figure 1.

\subsection{Investigating Potential Systematic Redshift Effects}

Quasar spectral studies have historically been plagued with difficulties in distinguishing between luminosity and redshift effects (see Steffen et al. 2006 for a recent example), particularly as spectral redshifting causes distinct spectral regions to be sampled in a given observed-frame bandpass.

In this study, the evident mid-infrared spectral curvature and the gap in the Spitzer photometric coverage between observedframe 8 and $24 \mu \mathrm{m}$ could induce apparent systematic differences in the mid-infrared spectra as a result of redshift. To examine this potential problem, we focused on a narrow redshift slice in our 


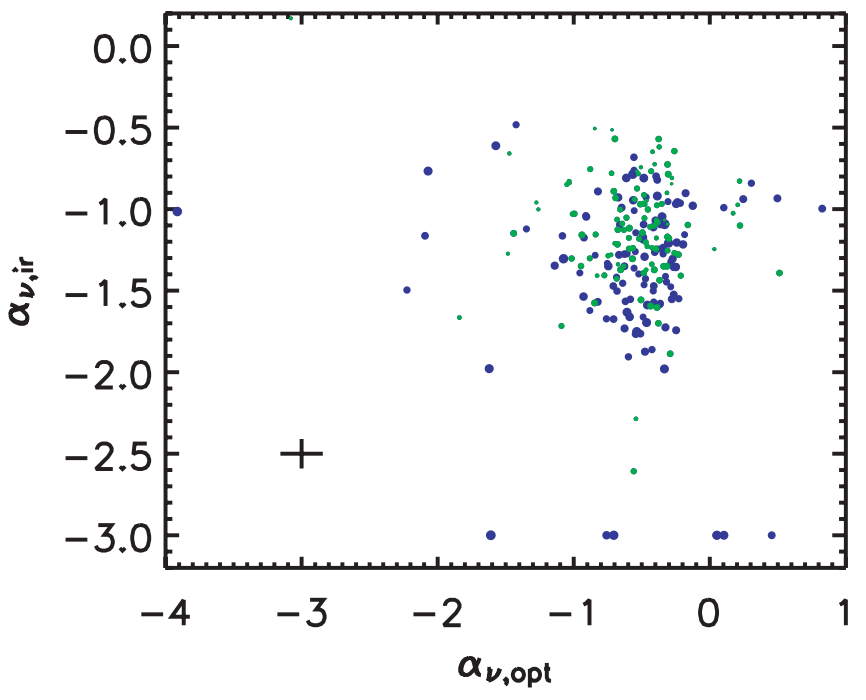

FIG. 7.- Infrared spectral index, $\alpha_{\nu \text {, IR }}$, vs. the optical spectral index, $\alpha_{\nu, \mathrm{opt}}$, for the entire SED sample. Quasars with only one photometric data point in the $1.8-8.0 \mu \mathrm{m}$ bandpass are plotted at $\alpha_{\nu \text { IR }}=-3.0$. The symbol sizes have been scaled to indicate the relative $L_{\mathrm{IR}}$ of each quasar; the larger symbols indicate more luminous quasars. For clarity, quasars with $\log \left(L_{\mathrm{IR}}\right)<46.0 \mathrm{ergs} \mathrm{s}^{-1}$ (the median of the $L_{\mathrm{IR}}$ distribution) have been colored green; more luminous quasars are blue. The plus sign in the lower left corner of the plot indicates the size of the median errors for the fitted values of $\alpha_{\nu, \text { IR }}$ and $\alpha_{\nu \text {, opt }}$.

sample, and reran the statistical tests of $\alpha_{\nu, \mathrm{IR}}$ versus $l_{\mathrm{IR}}$. We choose the redshift range from $z=1.0$ to 1.5 because there is still a significant ( $~(1 \mathrm{dex})$ infrared luminosity range, and at least three IRAC bands sample the rest-frame wavelength range of interest. This redshift subsample comprises 55 quasars. Even for this significantly smaller sample, the correlation of $\alpha_{\nu \text {, IR }}$ with $l_{\text {IR }}$ remains statistically significant, with both Spearman's and Kendall's tests giving $<1 \times 10^{-4}$ probabilities of no correlation. Furthermore, if the effect were entirely induced by bandpass effects, $\alpha_{\nu \text {, IR would }}$ be expected to correlate significantly with redshift, which is not the case (see Table 1). More even photometric sampling is likely needed to better constrain the shape of the correlation and certainly to investigate spectral curvature in greater detail.

\subsection{Comparing Optical and Infrared Spectral Indices}

In the standard understanding of the infrared emission in quasars, cold, dusty material on parsec scales from the supermassive black hole is illuminated by and reprocesses accretion disk radiation. The torus geometry is typically described as some form of flattened disklike configuration, encompassing toroids, open cones, and flared disks. At mid-infrared $(1-10 \mu \mathrm{m})$ wavelengths, the continuum spectrum is dominated by thermal dust emission, and as long as the highest dust temperature is fixed (by dust sublimation, for example), the SED is not sensitive to the spectral shape of the illuminating continuum regardless of the assumed geometry (Ivezic \& Elitzur 1997). Only at near-infrared $(<3 \mu \mathrm{m})$ wavelengths is the illuminating spectral shape expected to affect the SED as a result of scattering of direct continuum photons (M. Nenkova et al. 2007, in preparation). As seen in Figure 7, from these data we find no evidence for any correlation between $\alpha_{\nu \text {, opt }}$ and $\alpha_{\nu \text {, IR }}$, confirming theoretical expectation. Even when considering just the optically blue quasars to reduce the potential masking effects of optical extinction, the two parameters show no relation. Therefore, there is no evidence that the mid-infrared spectral changes are induced by the shape of the illuminating continuum; this is also consistent with the lack of notable differences

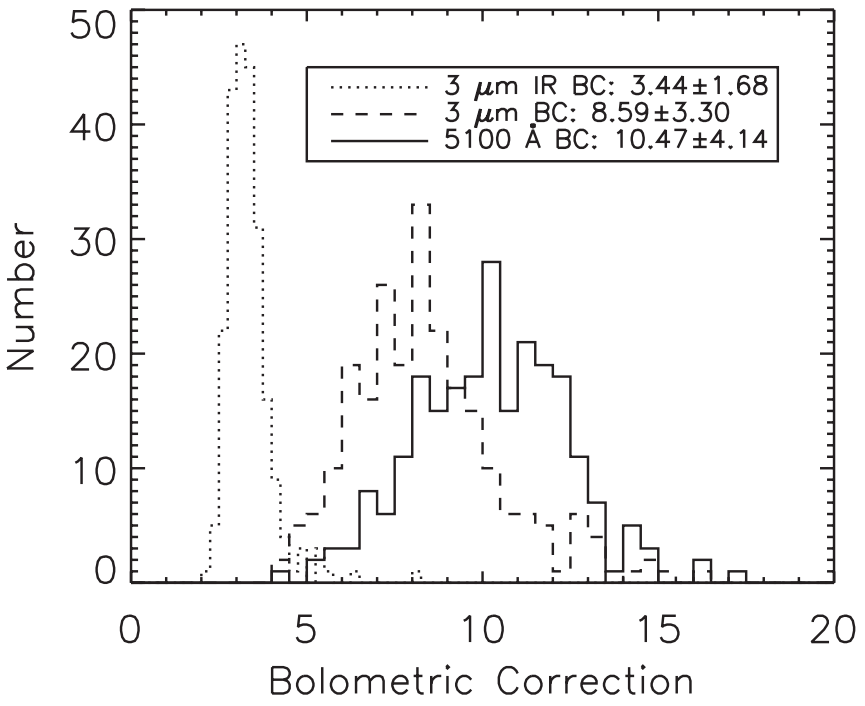

FIG. 8.-Distributions of BCs. The dotted histogram shows the corrections from $\nu L_{3 \mu \mathrm{m}}$ to $L_{\mathrm{IR}}$, the dashed histogram is the corrections from $\nu L_{3 \mu \mathrm{m}}$ to $L_{\mathrm{bol}}$, and the solid histogram represents the BCs from $\nu L_{5100 \AA}$ to $L_{\mathrm{bol}}$ as tabulated by R06a. The legend lists the mean and standard deviation of the mean for each distribution. The overall BCs from $\nu L_{3 \mu \mathrm{m}}$ show a comparable fractional dispersion to the standard (5100 ̊) BCs without being subject to dust extinction effects.

between the composite SEDs constructed by R06a from optically red and optically blue quasars.

\subsection{Evaluating Mid-Infrared and Optical Bolometric Corrections}

Typically, quasars only have photometric data in a few bandpasses, while the quantity of interest (e.g., for estimating black hole masses and Eddington accretion rates) is the integrated bolometric luminosity. Therefore, finding a single point in the SED from which a robust estimate of the bolometric luminosity can be made would be useful. Given the evidence discussed in $\S 2.1$ for the potentially significant effects of dust extinction on $l_{\text {opt }}$, we investigate whether a mid-infrared point would be more robust. Using the $3 \mu \mathrm{m}$ monochromatic luminosity, $\nu L_{3 \mu \mathrm{m}}$, we calculated both the overall (to $L_{\text {bol }}$ ) bolometric corrections (BCs) and the infrared (to $L_{\mathrm{IR}}$ ) BCs. The value of $L_{\mathrm{bol}}$ is integrated from $100 \mu \mathrm{m}$ to $10 \mathrm{keV}$; where photometry does not exist to measure this quantity, the composite SED was used to fill out the wavelength coverage. These are also compared to the typical, $5100 \AA$ $\mathrm{BCs}$ as compiled in R06a. Histograms of the three BC distributions are shown in Figure 8 . The $3 \mu \mathrm{m} \mathrm{BC}, 8.59 \pm 3.30$, is notably quite comparable to the $5100 \AA \mathrm{BC}, 10.47 \pm 4.14$, with fractional standard deviations of $38 \%$ and $40 \%$, respectively. The extremely tight and symmetric $3 \mu \mathrm{m}$ infrared BC indicates that $\nu L_{3 \mu \mathrm{m}}$ is quite a good proxy for $L_{\mathrm{IR}}$.

\section{DISCUSSION}

The distribution of $l_{\mathrm{IR}}$ versus $l_{\mathrm{opt}}$ as plotted in Figure $2 b$ is notably quite tight. As expected in a paradigm where the direct optical/UV continuum emission powers thermal emission in the infrared, optical, and infrared luminosity increase in tandem. While the overall sample of 234 quasars gives $L_{\mathrm{IR}} \propto L^{0.94 \pm 0.02}$, considering only the blue quasars (the least likely to be dustextincted) gives a linear relationship. This constant ratio of optical to infrared luminosities over $\sim 3$ decades in luminosity suggests that the dust mass (estimated from the infrared luminosity) radiating in the regime probed by Spitzer also increases approximately 
linearly with optical luminosity (assuming similar grain properties and distributions). At the same time, the inner radius of the dustemitting region increases as $\left(L_{\mathrm{UV}}\right)^{1 / 2}$, as the dust sublimation radius moves out. Thus, the inner wall of the torus at any luminosity is expected to have a similar temperature. It is challenging to extrapolate this point to inferences about the total infrared emitting volume, as for optically thick emission (expected for $\lambda<10 \mu \mathrm{m}$ ), the observed luminosity does not depend only on volume, but also on, for example, dust covering fraction, selfshadowing, and viewing angle. Therefore, it is not clear how our results are related to the interpretation that torus geometry must change with quasar power, as understood from the increasing type 1/type 2 ratio with luminosity found empirically in X-ray (Ueda et al. 2003; Akylas et al. 2006) and narrow emission-line surveys (Hao et al. 2005a).

Indications that quasar luminosity is affecting the torus structure are the significant spectral changes in the mid-infrared. Specifically, we seen an enhancement of mid-infrared emission in more luminous quasars manifested as a steepening of the $1.8-8.0 \mu \mathrm{m}$ continuum. As expected from the composite SEDs, this enhancement is better described as a bump, as an extrapolation from $\alpha_{\nu \text {, IR }}$ overpredicts the luminosity at $\sim 8 \mu \mathrm{m}$ in luminous quasars. In current static torus models, the shape of the mid-infrared continuum shortward of the $10 \mu \mathrm{m}$ silicate feature is sensitive to numerous physical inputs, including (1) orientation effects (e.g., Nenkova et al. 2002), (2) the dust grain properties (e.g., see Fig. 6 of Schartmann et al. 2005), (3) the clumpiness and number of emitting clouds (Dullemond \& van Bemmel 2005; M. Nenkova et al. 2007, in preparation), and (4) the opening angle of the torus (e.g., see Fig. 20 of Fritz et al. 2006). Our focus on type 1 quasars has reduced the effect of option (1) to the extent possible. Similarly, given that the inner wall of the torus is set by the graphite sublimation temperature to be $T \sim 1500 \mathrm{~K}$, a luminosity dependence of dust grain size, composition, and distribution is not expected, although detailed infrared spectroscopy is required to examine this issue empirically. Regarding option (3), current clumpy torus models do not generate explicit predictions for any luminosity dependence of the mid-infrared continuum. Therefore, option (4) seems plausible given the other evidence for a luminosity-dependent covering fraction of the torus. However, we are not aware of explicit predictions in the literature for how a changing opening angle would affect the mid-infrared continuum that are consistent with our results.

Another possible explanation for the enhanced mid-infrared spectra of luminous quasars is that a hotter dust component may be contributing more significantly to their SEDs than in lower luminosity objects. A striking example of this type of component was recently presented by Rodríguez-Ardila \& Mazzalay (2006) in the near-infrared spectrum of the narrow-line Seyfert 1 galaxy, Mrk 1239; they interpreted it as thermal emission from $T \sim$ $1200 \mathrm{~K}$ graphite grains. This type of spectral feature, the socalled near-infrared bump, has been noted for years (e.g., Hyland \& Allen 1982), and its physical origin was interpreted in the same fashion by Barvainis (1987). Similar structure is also evident in the mean radio-quiet composite spectrum of Elvis et al. (1994; although not, interestingly, in the radio-loud compositesee their Fig. 10), while the more recent combined SEDs of Hatziminaoglou et al. (2005) also show some hint of the nearinfared bump (see their Fig. 9). In fact, Edelson \& Malkan (1986) [who modeled the feature as a parabola peaked at $5.2 \mu \mathrm{m}$ in $\log \left(f_{\nu}\right)$ vs. $\log (\nu)$ units] found its strength to be correlated with luminosity in their study of 29 quasar SEDs. Given the IRAC wavelength sampling, at higher redshifts, the data are probing the shorter wavelengths of the mid-infrared bandpass, and the pres- ence of a "hot bump" peaking between 3 and $5 \mu \mathrm{m}$ would manifest itself as a steepening in $\alpha_{\nu \text {, IR }}$. The overprediction of $L_{8} \mu \mathrm{m}$ from extrapolations from the mid-infrared power law further supports this interpretation. This conclusion is not necessarily inconsistent with a "receding torus" paradigm, whereby more luminous quasars have tori covering less of the sky (Lawrence 1991), as a flatter, dusty structure would be less likely to block lines of sight to the hottest dust.

Finally, we also mention that polyaromatic hydrocarbon (PAH) emission is a possible contributor to the mid-infrared enhancement in more luminous quasars. We consider this unlikely, however, as the strongest PAH features (at 6.2 and $7.7 \mu \mathrm{m}$ ) are not sampled by the IRAC data beyond $z \sim 0.3$ and do not enter the MIPS $24 \mu \mathrm{m}$ bandpass until $z \sim 3$. Other PAH features in the $2-5 \mu \mathrm{m}$ range are much weaker in star-forming regions and laboratory spectra (Peeters et al. 2004), although they have not been well studied in luminous quasar spectra.

The gross similarity of quasar mid-infrared through optical SEDs is consistent with the finding of Brown et al. (2006) that both mid-infrared and optical type 1 quasar selection give similar space densities and luminosity functions for $z=1-5$. Therefore, although optical dust extinction may induce a false correlation as in Figure $2 a$, it is unlikely to distort strongly the study of type 1 quasars. Similar to our findings, a lack of variation of the ratio of far-infrared to optical luminosity as a function of bolometric luminosity was found by Andreani et al. (1999) for a sample of 120 quasars (with many far-infrared upper limits). However, we caution on the use of far-infrared $(\gtrsim 20 \mu \mathrm{m})$ luminosities to characterize quasars, as star formation in the host galaxy can contribute significantly to or even dominate the power in this regime. For example, from the $1-100 \mu \mathrm{m}$ spectral modeling of 36 type 1 quasars with luminosities matched to our sample $\left(L_{\text {quasar }}>3 \times\right.$ $10^{44} \mathrm{ergs} \mathrm{s}^{-1}$ ) presented in Fritz et al. (2006), the range of modeled quasar contributions to the total 5-1000 $\mu \mathrm{m}$ luminosities ranged from $9 \%$ to $100 \%$ with a mean of $62 \% \pm 26 \%$. The correlation between the far-infrared luminosities and $7.7 \mu \mathrm{m} \mathrm{PAH}$ emission seen by Schweitzer et al. (2006) in a sample of quasars and ultraluminous infrared galaxies further supports star formation as a significant (and variable) source of far-infrared power in luminous active galaxies. However, in typical torus + star formation model fits, the quasar dominates from $\sim 2$ to $10 \mu \mathrm{m}$ (e.g., van Bemmel \& Dullemond 2003), even considering some contribution from PAH emission. As the integrated $L_{\mathrm{IR}}$ values used in this work were derived from IRAC $+24 \mu \mathrm{m}$ MIPS data, they may more accurately represent the quasar power than infrared luminosities estimated from longer wavelength data.

\section{SUMMARY AND CONCLUSIONS}

We have analyzed 234 radio-quiet quasar SEDs from the sample of R06a with the goal of investigating possible midinfrared SED changes as a function of luminosity. While we find quasar SEDs overall to be remarkably stable with luminosity in this wavelength regime, there are some notable differences. We detail the following results:

1. Over three decades in infrared luminosity, the mean ratio and scatter of $L_{\mathrm{IR}} / L_{\mathrm{opt}}$ versus $L_{\mathrm{IR}}$ in quasar SEDs is constant. Some part of the spread in the distribution of $L_{\mathrm{IR}} / L_{\mathrm{opt}}$ is incurred from optical dust extinction. Given that dust extinction can significantly reduce $L_{\mathrm{opt}}$, the mid-infrared luminosity may be a more robust indicator of bolometric luminosity.

2. The steepening of the $1.8-8.0 \mu \mathrm{m}$ spectral index, $\alpha_{\nu \text {, IR }}$, with luminosity coupled with the spectral curvature evident from $L_{8} \mu \mathrm{m}$ measurements indicates that more luminous quasars 
show a mid-infrared enhancement consistent with a 3-5 $\mu \mathrm{m}$ bump. This result confirms the qualitative differences pointed out by R06a between luminous and faint composite quasar SEDs. As noted in previous studies, this feature can be attributed to an increasing contribution from hot $(T>1000 \mathrm{~K})$ dust with increasing quasar power (Barvainis 1987).

3. The overall bolometric correction from the monochromatic $3 \mu \mathrm{m}$ luminosity, $8.59 \pm 3.30$, has a comparable dispersion to the typical bolometric correction from $\nu L_{5100} \AA$ without suffering from dust extinction. The infrared bolometric correction from $\nu L_{3 \mu \mathrm{m}}$ to $L_{\mathrm{IR}}, 3.44 \pm 1.68$, shows a very tight and symmetric distribution, and is therefore a good measure of midinfrared power.

Although the first two results point strongly to a change in the geometry of the mid-infrared emitting region with luminosity, we know of no predictions from current torus models that are consistent with these findings. The notable $1.8-8.0 \mu \mathrm{m}$ spectral changes we have found therefore furnish strong constraints for future theoretical modeling of mid-infrared torus emission.

Static models for the torus are ultimately unsatisfying; our results support the picture where quasars are shaping the environment that emits in the mid-infrared - the "torus" is not simply a passive reprocessor of UV/optical emission. The dynamical wind paradigm, in which magnetohydrodynamic and radiation pressure lift and accelerate a dusty wind away from the central engine, is therefore more appealing (Königl \& Kartje 1994; Everett et al. 2002; Elitzur \& Shlosman 2006), and radiation pressure naturally has a luminosity dependence. Detailed predictions of mid-infrared spectra within this framework are a necessary next step, and future studies of $1-10 \mu \mathrm{m}$ infrared spectra of type 1 quasars matched in redshift with a range of luminosities will illuminate the nature of the spectral differences we find. Comparisons with theoretical models of such data will enable constraints on physical properties of interest such as the temperature, structure, and radius of the dusty region to aid in understanding the role of luminosity in shaping the quasar environment.

We thank Maia Nenkova, Matt Malkan, and Bev Wills for sharing their expertise. Support for S. C. G. was provided by NASA through the Spitzer Fellowship Program, under award 1256317. This research has made use of the Sloan Digital Sky Survey (http://www.sdss.org).

Facilities: Spitzer, Sloan
Akylas, A., Georgantopoulos, I., Georgakakis, A., Kitsionas, S., \& Hatziminaoglou, E. 2006, A\&A, 459, 693

Andreani, P., Franceschini, A., \& Granato, G. 1999, MNRAS, 306, 161

Avni, Y., \& Tananbaum, H. 1986, ApJ, 305, 83

Baldwin, J. A. 1977, ApJ, 214, 679

Barvainis, R. 1987, ApJ, 320, 537

Brown, M. J. I., et al. 2006, ApJ, 638, 88

Dullemond, C. P., \& van Bemmel, I. M. 2005, A\&A, 436, 47

Edelson, R. A., \& Malkan, M. A. 1986, ApJ, 308, 59

Elitzur, M., \& Shlosman, I. 2006, ApJ, 648, L101

Elvis, M., et al. 1994, ApJS, 95, 1

Everett, J., Königl, A., \& Arav, N. 2002, ApJ, 569, 671

Fritz, J., Franceschini, A., \& Hatziminaoglou, E. 2006, MNRAS, 366, 767

Haas, M., et al. 2003, A\&A, 402, 87

Hao, L., et al. 2005a, AJ, 129, 1795 2005b, ApJ, 625, L75

Hatziminaoglou, E., et al. 2005, AJ, 129, 1198

Hines, D. C., Krause, O., Rieke, G. H., Fan, X., Blaylock, M., \& Neugebauer, G. 2006, ApJ, 641, L85

Hopkins, P. F., et al. 2004, AJ, 128, 1112

Hyland, A. R., \& Allen, D. A. 1982, MNRAS, 199, 943

Isobe, T., Feigelson, E. D., Akritas, M. G., \& Babu, G. J. 1990, ApJ, 364, 104

Ivezic, Z., \& Elitzur, M. 1997, MNRAS, 287, 799

Jiang, L., et al. 2006, AJ, 132, 2127

Königl, A., \& Kartje, J. F. 1994, ApJ, 434, 446

Laor, A., \& Brandt, W. N. 2002, ApJ, 569, 641

Lawrence, A. 1991, MNRAS, 252, 586

\section{REFERENCES}

Nenkova, M., Ivezić, Ž., \& Elitzur, M. 2002, ApJ, 570, L9

Peeters, E., Allamandola, L. J., Bauschlicher, C. W., Jr., Hudgins, D. M., Sandford, S. A., \& Tielens, A. G. G. M. 2004, ApJ, 604, 252

Richards, G. T., Vanden Berk, D. E., Reichard, T. A., Hall, P. B., Schneider, D. P., SubbaRao, M., Thakar, A. R., \& York, D. G. 2002, AJ, 124, 1

Richards, G. T., et al. 2003, AJ, 126, 1131

. 2006a, ApJS, 166, 470 (R06a) 2006b, AJ, 131, 2766

Rodríguez-Ardila, A., \& Mazzalay, X. 2006, MNRAS, 367, L57

Schartmann, M., Meisenheimer, K., Camenzind, M., Wolf, S., \& Henning, T. 2005, A\&A, 437, 861

Schneider, D. P., et al. 2005, AJ, 130, 367

Schweitzer, M., et al. 2006, ApJ, 649, 79

Shang, Z., Wills, B. J., Robinson, E. L., Wills, D., Laor, A., Xie, B., \& Yuan, J. 2003, ApJ, 586, 52

Shemmer, O., et al. 2006, ApJ, 644, 86

Siebenmorgen, R., Haas, M., Krügel, E., \& Schulz, B. 2005, A\&A, 436, L5

Spergel, D. N., et al. 2003, ApJS, 148, 175 2007, ApJ, in press (astro-ph/0603449)

Steffen, A. T., Strateva, I., Brandt, W. N., Alexander, D. M., Koekemoer, A. M., Lehmer, B. D., Schneider, D. P., \& Vignali, C. 2006, AJ, 131, 2826

Strateva, I. V., Brandt, W. N., Schneider, D. P., Vanden Berk, D. G., \& Vignali, C. 2005, AJ, 130, 387

Ueda, Y., Akiyama, M., Ohta, K., \& Miyaji, T. 2003, ApJ, 598, 886

van Bemmel, I. M., \& Dullemond, C. P. 2003, A\&A, 404, 1

Vanden Berk, D. E., et al. 2001, AJ, 122, 549

York, D. G., et al. 2000, AJ, 120, 1579 